\title{
Sensitivity "Hot Spots" in the Direct Analysis in Real Time Mass Spectrometry of Nerve Agent Simulants
}

\author{
Glenn A. Harris, Caitlin E. Falcone, Facundo M. Fernández \\ School of Chemistry and Biochemistry, Georgia Institute of Technology, Atlanta, GA 30332, USA
}

\begin{abstract}
Presented here are findings describing the spatial-dependence of sensitivity and ion suppression effects observed with direct analysis in real time (DART). Continuous liquid infusion of dimethyl methyl phosphonate (DMMP) revealed that ion yield "hot spots" did not always correspond with the highest temperature regions within the ionization space. For instance, at lower concentrations $(50$ and $100 \mu \mathrm{M})$, the highest sensitivities were in the middle of the ionization region at $200{ }^{\circ} \mathrm{C}$ where there was a shorter ion transport distance, and the heat available to thermally desorb neutrals was moderate. Conversely, at higher DMMP concentrations $(500 \mu \mathrm{M})$, the highest ion yield was directly in front of the DART source at $200{ }^{\circ} \mathrm{C}$ where it was exposed to the highest temperature for thermal desorption. In matching experiments, differential analyte volatility was observed to play a smaller role in relative ion suppression than differences in proton affinity and the relative sampling positions of analytes. At equimolar concentrations sampled at the same position, suppression was as high as $26 \times$ between isoquinoline (proton affinity $952 \mathrm{~kJ} \mathrm{~mol}^{-1}$, boiling point $242{ }^{\circ} \mathrm{C}$ ) and $p$-anisidine (proton affinity $900 \mathrm{~kJ} \mathrm{~mol}^{-1}$, boiling point $243^{\circ} \mathrm{C}$ ). This effect was exacerbated when sampling positions of the two analytes differed, reaching levels of relative suppression as high as $4543.0 x \pm 1406$.0. To mitigate this level of relative ion suppression, sampling positions and molar ratios of the analytes were modified to create conditions in which ion suppression was negligible.
\end{abstract}

Key words: Direct analysis in real time (DART), Ambient MS, Ionization fundamentals, Ion suppression, Nerve agent simulants

\section{Introduction}

$\mathrm{T}$ he growth of the ambient desorption/ionization mass spectrometry (MS) field has been rapid, with various different ionization techniques and corresponding applications being reported in a short time span [1-3]. Ambient MS

Electronic supplementary material The online version of this article (doi:10.1007/s13361-011-0276-8) contains supplementary material, which is available to authorized users.

Correspondence to: Facundo M. Fernández; e-mail: facundo.fernandez@ chemistry.gatech.edu techniques enable: (1) surface ionization in the absence of enclosures, (2) direct ionization with minimum sample pretreatment, (3) interfacing to most types of mass spectrometers fitted with differentially-pumped atmospheric pressure interfaces, and (4) soft ion generation (low internal energy deposition) such that the majority of detected ions are intact. A subset of techniques in the ambient MS field revolves around direct and alternating current (DC and $\mathrm{AC}$ ) plasma-based approaches involving chemical ionization (CI) mechanisms. Although several ambient plasma-based ionization techniques have been developed since the introduction of direct analysis in real time (DART) [4], DART remains the most prevalently studied and used ambient 
plasma ionization technique due to its commercial availability with over 70 published references within the past two years alone.

Despite the wide adoption of DART as a high-throughput screening tool for pharmaceuticals [5-7], homeland security [8-11], metabolomics [12,13], and polymer analysis [14, 15], the fundamental variables that affect DART analysis are still under investigation. The ionization region in DART MS is a dynamic environment involving complex fluid dynamics, steep temperature gradients, and weak electrostatic fields that are a result of the ion source geometry, sample position and orientation, and instrumental settings [16]. In situations where only qualitative information is required, spatial effects can be largely ignored. However, when highly reproducible and/or quantitative experiments are desired, sample positioning becomes critical to ensure reproducibility of the ionization conditions. Ionization may proceed through several atmospheric pressure reaction pathways involving the primary reactive species formed by DART (metastables) and secondary reactive species such as protonated species from atmospheric gases. The factors that affect the prevailing ionization pathway depend on the presence and type of sample matrix, solvents and contaminants, the DART gas composition, and the chemical properties of the analyte [17]. Even with optimized parameters, there is some evidence that DART may sometimes succumb to deleterious matrix effects involving ion suppression [18] when the depletion point of reactive species has been reached [19]. Ion suppression is expected to be present to some extent in all ion direct generation approaches, and affect sensitivity and dynamic range by preferentially decreasing analyte signals. The major DART conditions to consider when mitigating ion suppression include the rates of neutral desorption (related to the temperature and flow rate of the DART gas and analyte boiling point, BP), ionization efficiency (proton affinity, PA), and molar ratios of the analytes of interest respect to interfering species. These effects are confounded with the sampling position and orientation in space. From our past experience with DART, we believe that the desorption and ionization processes have differential magnitudes relative to the sample position in space resulting in convoluted ion intensities and relative levels of ion suppression $[13,16]$. To study what physicochemical processes and DART conditions contribute to sensitivity, ionization efficiency, ion suppression, and dynamic range, a series of steady-state spatially-resolved experiments were devised to study these effects in more detail.

\section{Experimental}

\section{Reagents}

All reagents were analytical grade (Sigma-Aldrich, St. Louis, MO, USA) and used without further purification. Solutions of dimethyl methylphosphonate (DMMP, 97\%), $p$-anisidine (99\%), and isoquinoline (97\%) were prepared in nanopure water (Barnstead International, Dubuque, IA, USA). Ultra high purity helium (99.999\%; Airgas, Atlanta, GA, USA) was used for the DART glow discharge gas.

\section{Instrumentation}

MS analysis was performed with a commercial DART-100 ionization source (IonSense, Inc., Saugus, MA, USA) coupled to a quadrupole-time of flight (Q-TOF) mass spectrometer (Bruker micrOTOF-Q I; Bremen, Germany). The Q-TOF mass spectrometer interface was modified to accommodate for the gas flow of the DART source through the addition of a custom gas-ion separator tube (GIST; IonSense Inc,. Saugus, MA, USA) connected to a Vacuubrand $2 \mathrm{C}$ diaphragm pump (Vacuubrand, Wertheim, Germany). The DART source was operated with helium at $1 \mathrm{Lmin}^{-1}$ at a gas temperature of $100-400{ }^{\circ} \mathrm{C}$, discharge voltage of $3500 \mathrm{~V}$ and a grid electrode voltage of $50 \mathrm{~V}$. The particular flow rate used in this study was chosen because it is the maximum allowed by the mass spectrometer. The interface includes a pre-pumped gas ion separator tube (GIST) to reduce the vacuum load on the first differentially pumped region of the instrument. However, even a small rise in helium flow rate (to $1.2 \mathrm{Lmin}^{-1}$ ) raises that stage's pressure beyond the safe operating conditions and automatically shuts down the power supplies providing the ion optics potentials. The mass spectrometer settings were as follows: end plate offset $-500 \mathrm{~V}$, capillary $-2000 \mathrm{~V}$, dry gas (nitrogen) $2 \mathrm{Lmin}^{-1}$, dry gas temperature $150{ }^{\circ} \mathrm{C}$, spectra acquired at $1 \mathrm{~Hz}$ in the $50-1000 \mathrm{~m} / \mathrm{z}$ range.

\section{Experimental Set-up}

DART MS sensitivity, dynamic range and ion suppression experiments were performed via continuous liquid sampling to ensure a stable DART ion signal. Although this is not a traditional sampling method for DART MS, other sampling approaches such as dried droplet analysis on a sampling probe, a solid sample, or suspended liquid can suffer from sampling inaccuracies, even when automated. Maximum reproducibility in the signal was necessary to enable mapping sensitivity effects accurately. For this, a custom nylon sample holder was machined to ensure sampling reproducibility at discrete positions (Fig. 1). The sample holder contained nine sampling holes arranged in a $3 \times 3$ grid with a $6.5 \mathrm{~mm}$ separation across rows $1-3$ and $4.5 \mathrm{~mm}$ across columns A-C. The sampling grid was positioned so that column B was in direct alignment with the DART gas nozzle and the GIST. Each spot in space was probed by introducing a continuous flow $\left(600 \mu \mathrm{L} \mathrm{h}-{ }^{1}\right)$ of sample through a stainless steel capillary (o.d. $450 \mu \mathrm{m}$, i.d. $305 \mu \mathrm{m}$ ) inserted through the bottom of the sample holder. This capillary was grounded to prevent charging by the continuous bombardment with ions. To prevent the stainless steel capillary from oscillating in the DART gas stream, a glass capillary protected the outside of the sampling line by 


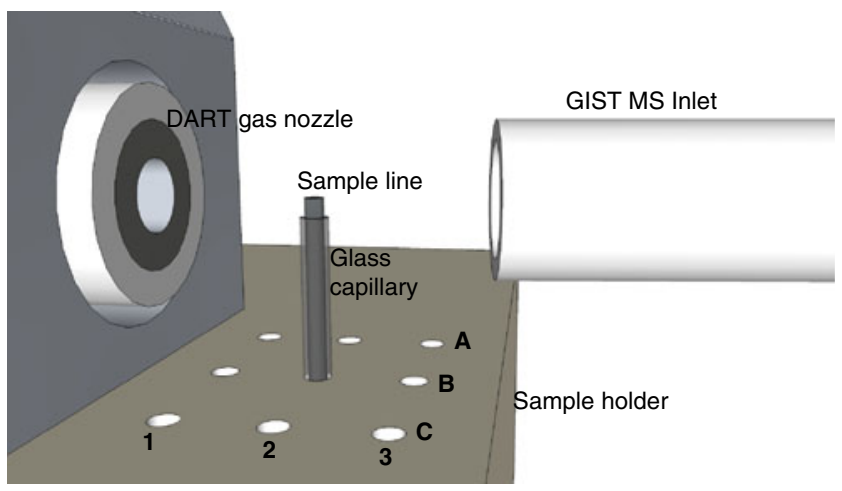

Figure 1. DART MS ionization region depicting the DART gas nozzle, GIST sampling tube to the mass spectrometer inlet, and the sample grid with the sampling probe in the B2 position. Sampling distances between each spot along rows 1-3 was $6.5 \mathrm{~mm}$ and $4.5 \mathrm{~mm}$ along columns $\mathrm{A}-\mathrm{C}$

providing a rigid base for support. The stainless steel sample line extended $1 \mathrm{~mm}$ above the glass capillary and was aligned with the center of the DART gas nozzle outlet. This position ensured maximum coverage of the sampling probe with the reactive gas. Steady-state experiments were recorded for all measurements. For each experiment, data was collected in a single day to ensure reproducibility except in the case of the sensitivity-in-space and dynamic range trials. Due to the length of time needed to conduct these experiments, day-to-day quality control runs were collected at set concentrations and positions showing that a minimum $\pm 10 \%$ reproducibility (Supplementary Figure S1) could be achieved. In all experiments, the average signal (protonated molecule in all cases as no dimers, adducts or fragments were detected) was acquired for five 1-min segments at each specified DART condition and spot-in-space. The average of all five acquired segments was reported in all cases below. Whenever sample concentrations were changed, the stainless steel capillary was thoroughly rinsed with pure methanol, 50/50 methanol-water and pure water three times. The line was reattached with pure water running through the line and the DART source on to insure no sample carryover was observed. Temperature mapping of the ionization region was determined with an $80 \mathrm{BK}$ temperature probe connected to a digital multimeter (Fluke 179-True RMS; Everett, WA, USA). All data were processed using Sigma Plot 10.0 (Systat Software Inc., San Jose, CA, USA). All proton affinity values used are those at room temperature.

\section{Results and Discussion}

\section{Sampling Region Temperature Gradient}

Previous observations have shown there is a steep temperature gradient along the line connecting the DART gas nozzle to the mass spectrometer inlet $[16,20]$. However, given that sample surface areas may extend beyond the center-line of the ionization region, the temperature of the relative sensitivities in the entire ionization region were now mapped (Fig. 2) through peripheral sampling spots (columns $\mathrm{A}$ and $\mathrm{C}$, Fig. 1). For all DART temperature settings, the hottest region was in the center-line (column B) aligned with the DART gas nozzle. The extent of temperature drop-off between the nozzle (sample spot B1) and the GIST MS inlet (sample spot B3) was $29.9^{\circ} \mathrm{C}(1,2,3: 195.0 \pm 3.1,176.4 \pm$
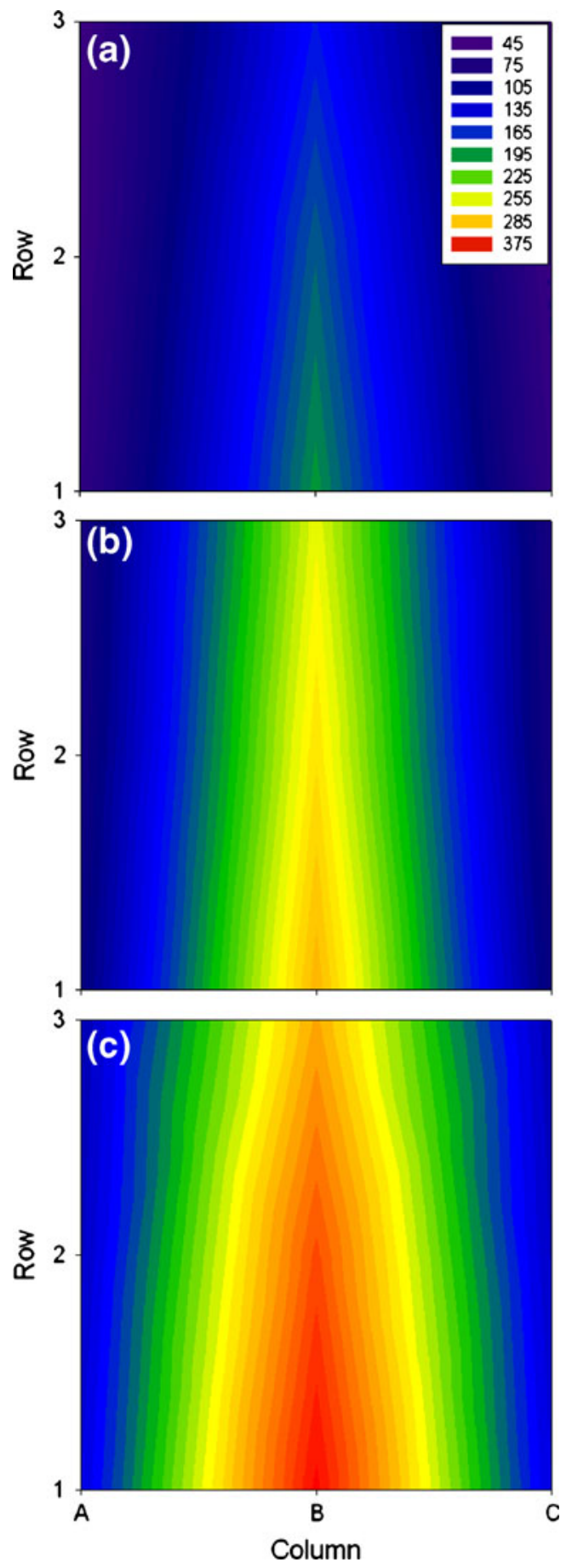

Figure 2. Direct probe temperature maps of the DART ionization region at gas temperatures of (a) $200{ }^{\circ} \mathrm{C}$, (b) $300{ }^{\circ} \mathrm{C}$ and (c) $400{ }^{\circ} \mathrm{C}$. The color scale spans the temperature range measured across all three software temperature settings 
$7.5,165.1 \pm 1.2^{\circ} \mathrm{C}$ ) with a software set temperature of $200{ }^{\circ} \mathrm{C}$, $35.0{ }^{\circ} \mathrm{C}\left(1,2,3: 285.0 \pm 1.9,270.1 \pm 1.8,250.0 \pm 2.3{ }^{\circ} \mathrm{C}\right)$ with a software temperature setting of $300{ }^{\circ} \mathrm{C}$ and $50.1{ }^{\circ} \mathrm{C}(1,2,3$ : $\left.365.1 \pm 3.4,338.4 \pm 2.1,315 \pm 2.0^{\circ} \mathrm{C}\right)$ with a software temperature setting of $400{ }^{\circ} \mathrm{C}$ (Fig. 2a, b, and c, respectively). In the lateral sampling regions (columns $\mathrm{A}$ and $\mathrm{C}$ ), the temperature decrease was less than at the center (column B), but overall temperatures were much lower. When the software temperature setting was $200{ }^{\circ} \mathrm{C}$, the temperature drop-off was only $11.0{ }^{\circ} \mathrm{C}\left(1,2,3: 56.0 \pm 1.9,48.1 \pm 0.4,45.0 \pm 0.7{ }^{\circ} \mathrm{C}\right)$ for column A and $12.2^{\circ} \mathrm{C}(1,2,3: 56.2 \pm 2.0,48.5 \pm 1.6,44.0 \pm$ $1.8^{\circ} \mathrm{C}$ ) for column $\mathrm{C}$ (Fig. 2a). The temperature drop-off was $16.0{ }^{\circ} \mathrm{C}\left(1,2,3: 92.1 \pm 3.3,81.7 \pm 0.7,76.1 \pm 1.8{ }^{\circ} \mathrm{C}\right)$ for column $\mathrm{A}$ and $14.3{ }^{\circ} \mathrm{C}(1,2,3: 91.0 \pm 3.0,80.2 \pm 0.4,76.7 \pm$ $2.0^{\circ} \mathrm{C}$ ) for column $\mathrm{C}$ when the temperature setting was set to $300{ }^{\circ} \mathrm{C}$ (Fig. 2b). When the temperature setting was $400{ }^{\circ} \mathrm{C}$, the temperature drop-off was $20.0{ }^{\circ} \mathrm{C}(1,2,3: 134.8 \pm 3.7$, $\left.122.4 \pm 2.2,114.8 \pm 2.7^{\circ} \mathrm{C}\right)$ for column $\mathrm{A}$ and $18.4^{\circ} \mathrm{C}(1,2,3$ : $134.4 \pm 3.0,123.2 \pm 1.9,116.0 \pm 2.1^{\circ} \mathrm{C}$ ) for column C (Fig. 2c). Although the overall temperatures in the lateral columns are much lower than in the central column B, the recorded temperature ranges in columns $\mathrm{A}$ and $\mathrm{C}$ were fairly identical. This result indicates a symmetric temperature contour map of the ionization region where little to no difference in thermal desorption efficiency should be observed between columns A and C.

\section{Spatial Sensitivity and Dynamic Range}

The symmetric temperature field observed in previous experiments supports the measured spatial sensitivity changes measured for DMMP, with some interesting additional effects (Fig. 3). Overall, the regions in space that yielded the highest ion signal were located along column B where the highest temperatures and, therefore, the most effective thermal desorption was present. At lower concentrations $(50$ and $100 \mu \mathrm{M})$, the most sensitive spot in space to sample from was B2 regardless of set DART gas temperature (Fig. 3a, b, d, e, g, and h). Interestingly, this spot does not correspond to the highest measured temperature (Fig. 2). B1, the hottest sampled spot, had a $40 \%-60 \%$ lower relative intensity compared with B2 at these lower DMMP concentrations. This suggests that at lower concentrations, it may be beneficial to sample in the middle of the ionization region to also enhance ion transport. As noted in previous studies [16], the high DART gas velocity and net vertical particle flows in the ionization region may potentially result in sample being transported away from the mass spectrometer inlet, decreasing the concentration of neutrals/ions in the ionization region. If sampling near the gas nozzle at B1, the analyte will be in the region of highest temperature but also of highest gas velocity. At this position, even if more total analyte is desorbed and ionized than B2, the higher gas velocity may blow neutrals and ions away from the inlet. Following this logic, it may seem best to sample at B3 directly in front of the GIST MS inlet to maximize ion transport into the instrument. However, B3 presented the lowest sensitivity by $\sim 80 \%$ compared with B2, and $40 \%$ compared with B1. Row 3 was the region that suffered from the lowest measured temperature across any sampling column translating to the lowest amount of thermallydesorbed neutrals. Coupled to this effect was the suction from the GIST MS inlet. Although inlet suction offsets neutral dilution by the DART gas, the rapid transit inside of the GIST MS inlet likely reduced the time for proton-transfer ionization reactions resulting in lower sensitivity.

The sensitivity map differed at higher sampled concentrations $(500 \mu \mathrm{M})$ with the highest sensitivity measured at position B1 (Fig. 3c, f, and i). These sensitivity maps corresponded well with the observed temperature gradients (Fig. 2) and suggest that dilution from the DART gas was not as significant of an effect. At such high concentration levels, sensitivity more closely follows the relative rates of thermal desorption throughout the sampling grid. Sensitivity changes were more pronounced at lower temperatures where the increase in relative intensity at B1 compared with B2 was $80 \%$ and $100 \%$ for DART gas temperatures of 200 and $300{ }^{\circ} \mathrm{C}$, respectively (Fig. $3 \mathrm{c}$ and f). At these two settings, the temperature drop-off causes the effective gas temperature to become much closer to the DMMP BP $\left(181^{\circ} \mathrm{C}\right)$, reducing the thermal desorption rate. At a temperature setting of $400^{\circ}$ C, the increase in intensity from B1 to B2 was only $40 \%$ due to the temperature still being much higher along the $\mathrm{B}$ sampling column (Fig. 3i).

It is also important to point out that sensitivities were $\sim 80 \%-$ $90 \%$ lower in any row along columns $\mathrm{A}$ and $\mathrm{C}$ compared with column B. The two major factors causing the low sensitivity were the lower overall temperatures at these positions and poor ion transmission trajectories towards the inlet. Temperatures were lower by $\sim 60 \%-80 \%$ on the outer columns $\mathrm{A}$ and $\mathrm{C}$ compared with column B (Fig. 2). Also, the sample positions along columns $\mathrm{A}$ and $\mathrm{C}$ were off axis to the GIST inlet. If ionized, the low quantity of desorbed molecules would have a more indirect and longer trajectory to the inlet. A possible means to further improve ion sampling would be to use a flared capillary inlet with a large entrance aperture at the opening of the GIST tube.

Following these experiments, DMMP was sampled in all three positions (B1, B2, B3) and gas temperatures $(200,300$, and $\left.400{ }^{\circ} \mathrm{C}\right)$ over a wider concentration range $(25 \mu \mathrm{M}$ to $1 \mathrm{mM}$ ) to assess the available dynamic range (Supplementary Figure S2). As a whole, three decades of concentration were detected with corresponding increases in signal response. Concentrations above $1 \mathrm{mM}$ were not used due to laboratory safety reasons although judging from the observe intensities, concentrations near 10-25 mM should be detected with similar trends. Throughout all tested variables, there was a decrease in overall sensitivity with an increase in set DART gas temperature (Supplementary Figure S2). This alludes to carefully tuning the rate of desorption to be sufficient enough to evaporate excess solvent (water), but not too high to induce fragmentation 

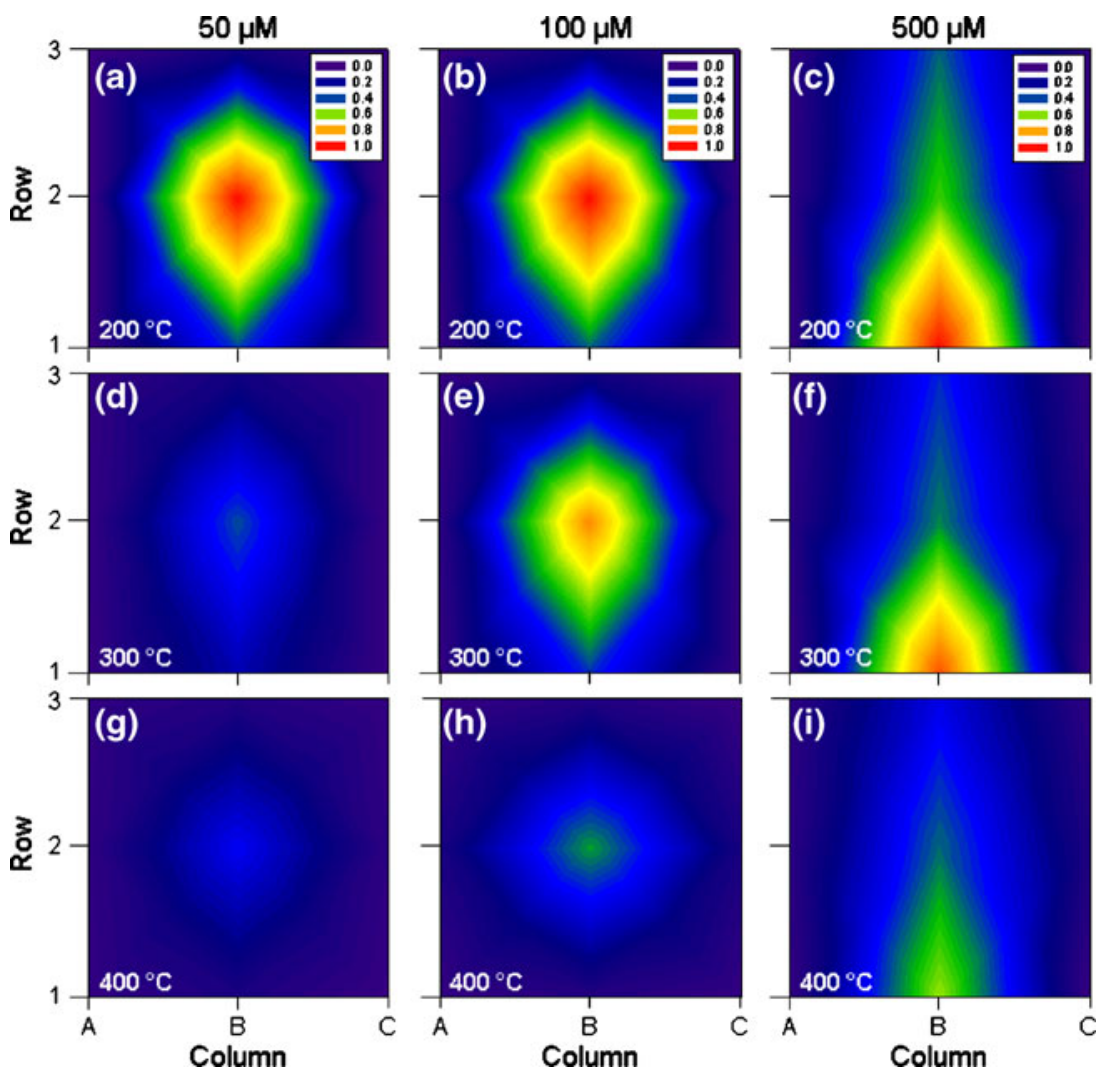

Figure 3. Sensitivity-in-space maps at different DART gas temperatures and concentrations of DMMP. Maps (a) $50 \mu \mathrm{M}$, (b) $100 \mu \mathrm{M}$, and (c) $500 \mu \mathrm{M}$ were tested at $200{ }^{\circ} \mathrm{C}$, maps (d) $50 \mu \mathrm{M}$, (e) $100 \mu \mathrm{M}$, and (f) $500 \mu \mathrm{M}$ were tested at $300{ }^{\circ} \mathrm{C}$, and maps (g) $50 \mu \mathrm{M}$, (h) $100 \mu \mathrm{M}$, and (i) $500 \mu \mathrm{M}$ were tested at $400^{\circ} \mathrm{C}$. All averaged $(n=5)$ intensities were normalized to the highest intensity recorded for a given concentration

and/or produce analyte neutrals faster than the reaction with protonated reactive species. However, this may be a minor effect that compounds with a lower volatility, such as higher molecular weight molecules, which may not experience due to the requirement for additional heat to be thermally desorbed.

Overall the B3 position corresponded to the poorest overall sensitivity at any tested concentration level due to poor thermal desorption. However, this position provided the highest linearity. The most discernable changes in signal intensity with respect to concentration was observed for B3 (Supplementary Figure S2a). Extension of the sampled concentrations to probe the linear dynamic range $(1-100 \mu \mathrm{M})$ showed strong linearity at B3 at all three temperatures $\left(200{ }^{\circ} \mathrm{C}: \mathrm{r}^{2}=0.981,300{ }^{\circ} \mathrm{C}\right.$ : $\mathrm{r}^{2}=0.975$, and $400{ }^{\circ} \mathrm{C}: \mathrm{r}^{2}=0.985$ ) (Supplementary Figure $\mathrm{S} 3 \mathrm{a})$. At lower concentrations $(25,50,75$, and $100 \mu \mathrm{M})$, the middle position, B2 had the highest sensitivity supporting the previous results (Supplementary Figure $\mathrm{S} 2 \mathrm{~b})$. The linear dynamic range for B2 at 200, 300, and $400{ }^{\circ} \mathrm{C}$ was lower than at B3 with $\mathrm{r}^{2}=0.940,0.876$, and 0.880 , respectively (Supplementary Figure S3b). Position B1 also corresponded well to the previous sensitivity map showing the highest sensitivity for the highest tested concentrations $(250 \mu \mathrm{M}, 500 \mu \mathrm{M}$, and $1 \mathrm{mM}$, Supplementary
Figure S2c). The linear dynamic range for 200, 300, and $400{ }^{\circ} \mathrm{C}$ was lower than at B3 with $\mathrm{r}^{2}=0.911,0.781$, and 0.980 , respectively (Supplementary Figure S3c). All these data suggest that future routine sensitive quantitative or semiquantitative DART methods would likely see advantageous results by positioning samples in regions where both the role of suction to improve ion transmission and localized heating are maximized.

\section{Ion Suppression}

A series of competitive ionization reactions were carried out along column B to determine the effect of analyte BPs and $\mathrm{PA}$ on the observed relative intensities as a function of sample position. For the first set of experiments, an equimolar solution of DMMP (PA: $902 \mathrm{~kJ} \mathrm{~mol}^{-1}$, BP: $\left.181{ }^{\circ} \mathrm{C}\right)[21]$ and $p$-anisidine (PA: $900 \mathrm{~kJ} \mathrm{~mol}^{-1}$, BP: $243{ }^{\circ} \mathrm{C}$ ) [22] was tested to determine whether volatility of analytes at different temperatures and sampling positions contributed to suppression. When the DART set temperature was below the BPs of both compounds $\left(100{ }^{\circ} \mathrm{C}\right)$, there was little observed suppression at low concentrations $(50 \mu \mathrm{M})$ regardless of the sample position, since only very small amounts of either analyte were thermally desorbed (Fig. 4a). Suppression increased with increasing concentration due to competition 
(a)

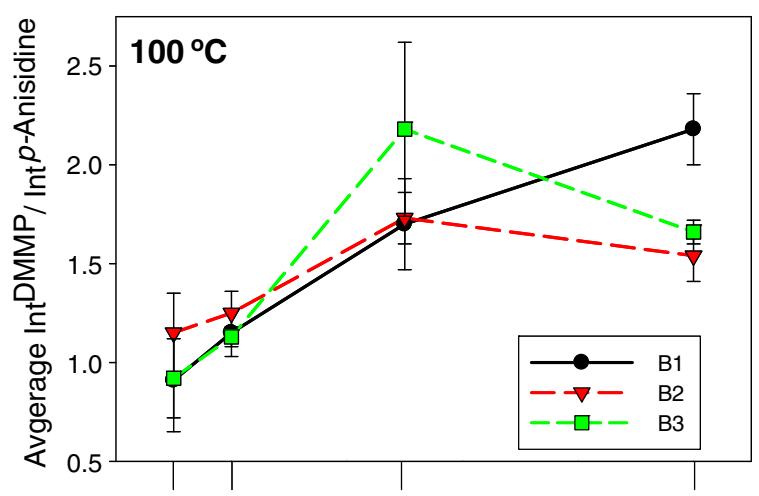

(c)

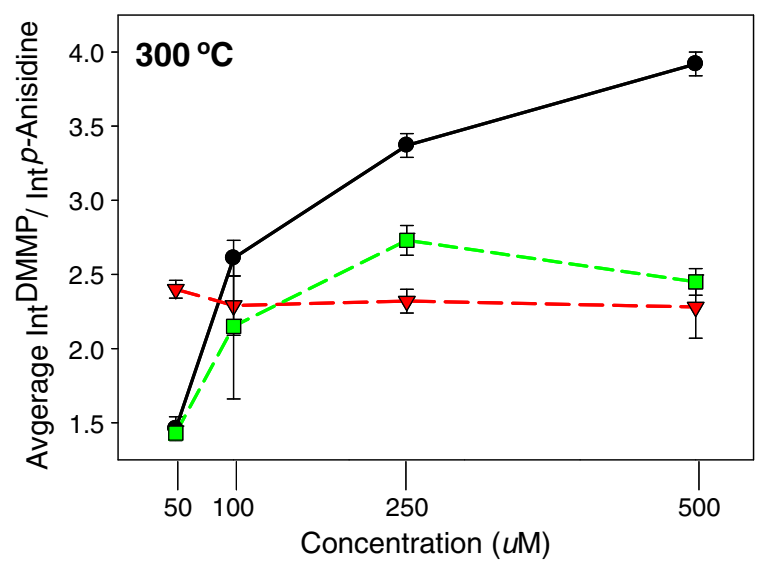

(b)

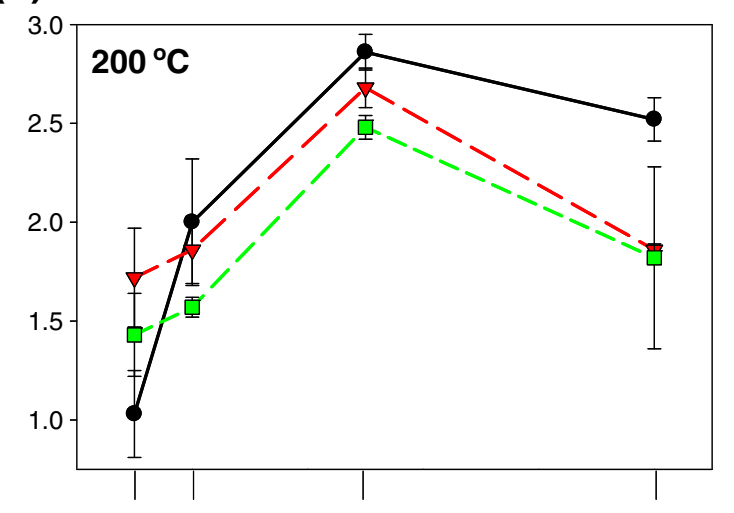

(d)

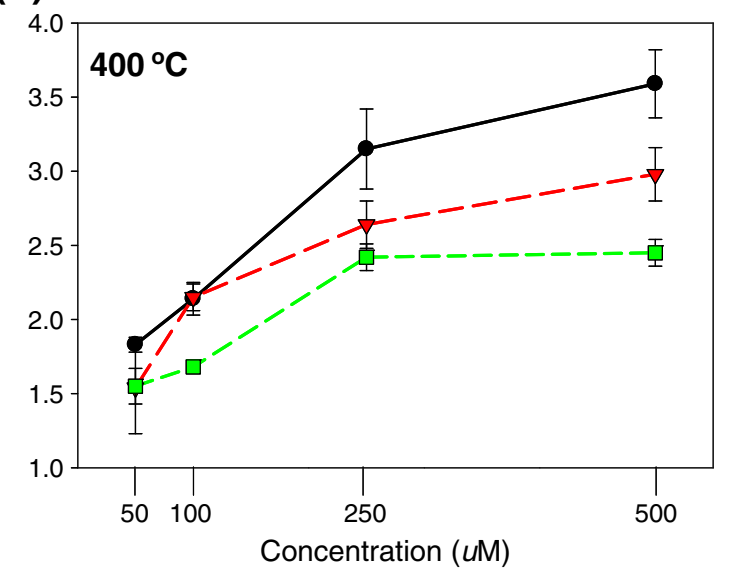

Figure 4. Effect of different boiling points but similar proton affinity on ion suppression at different sampling spots of equimolar mixtures of DMMP (BP: $181^{\circ} \mathrm{C}$, PA $902 \mathrm{~kJ} \mathrm{~mol}^{-1}$ ) and $p$-anisidine (BP: $243{ }^{\circ} \mathrm{C}, \mathrm{PA}: 900 \mathrm{~kJ} \mathrm{~mol}^{-1}$ ) at DART gas temperatures of: (a) $100{ }^{\circ} \mathrm{C}$, (b) $200{ }^{\circ} \mathrm{C}$, (c) $300{ }^{\circ} \mathrm{C}$ and (d) $400{ }^{\circ} \mathrm{C}$

for protonated water clusters in the reaction region, as expected. Even in a low temperature environment, the compound with the lowest BP (DMMP) suppressed the compound with a higher BP ( $p$-anisidine). This trend was more pronounced at higher DART temperatures (Fig. 4b, c, and d). At the lowest concentration $(50 \mu \mathrm{M})$ and at temperatures of $200-400{ }^{\circ} \mathrm{C}, 1.1$ - to 2.4 -fold suppression was observed, regardless of the sample position. There was no clear trend regarding the effect of position on suppression at low concentrations (Fig. 3). However at higher concentrations $(100-500 \mu \mathrm{M})$, suppression was generally more pronounced in the $\mathrm{B} 1$ position $(2.0-3.8 \times)$ across all temperatures in the $200-400{ }^{\circ} \mathrm{C}$ interval. Position B1 also showed the best sensitivity at high concentrations (Fig. 3c, f, and i), suggesting the region is rich with reactive species, and ion losses during transit were compensated by the overall higher rate of protonation. Suppression did not vary too much between positions $\mathrm{B} 2$ and $\mathrm{B} 3$ at higher concentrations, with position B2 having slightly higher suppression at 200 and $400{ }^{\circ} \mathrm{C}$. Regardless, both positions produced lower suppression in the higher concentration range compared with B1 due to their poorer overall sensitivity, indicative of a smaller population of desorbed neutrals, which decreased suppression effects.

An identical experiment was carried out between isoquinoline (IsoQ, PA $952 \mathrm{~kJ} \mathrm{~mol}^{-1}$, BP $242{ }^{\circ} \mathrm{C}$ ) [22] and $p$-anisidine (PA: $900 \mathrm{~kJ} \mathrm{~mol}^{-1}$, BP: $243{ }^{\circ} \mathrm{C}$ ), where analyte BPs were almost equal but PAs were significantly different. Figure 5 demonstrates that observed levels of ion suppression for analytes at equimolar concentrations were temperature, position and concentration dependent. For all three tested temperatures $\left(200,300\right.$, and $\left.400{ }^{\circ} \mathrm{C}\right)$, the average suppression was always greater than in Fig. 4. As a whole, the suppression observed at $200{ }^{\circ} \mathrm{C}$ was greater at all positions than at 300 and $400{ }^{\circ} \mathrm{C}$, indicating that proton exchange is more favorable for higher PA analytes at lower temperatures. This may be due to the lower thermodynamic stability of smaller protonated water clusters at lower temperatures, a phenomenon that has been observed with both DART [20] and APCI [23]. At $200{ }^{\circ} \mathrm{C}$, ion suppression for position B1 ranged from 6 to $26 \times$ in the tested concentration range $(50-500 \mu \mathrm{M})$, and was similar for positions $\mathrm{B} 2$ and $\mathrm{B} 3$, ranging from 7 to $19 \times$ (Fig. 5a). For both $300{ }^{\circ} \mathrm{C}$ and $400{ }^{\circ} \mathrm{C}$, measured ion suppression did not vary much, ranging for $\mathrm{B} 1$ from 5 to $17 \times$ and for $\mathrm{B} 2$ and $\mathrm{B} 3$ 
(a)

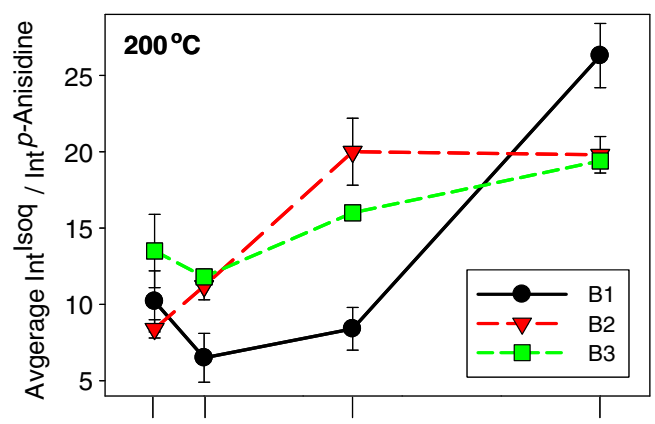

(b)

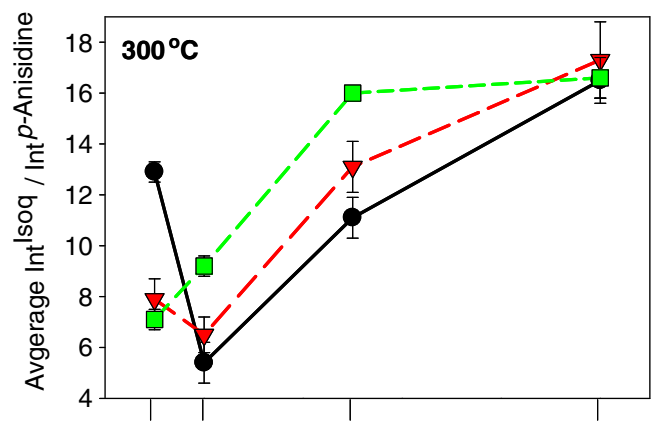

(c)

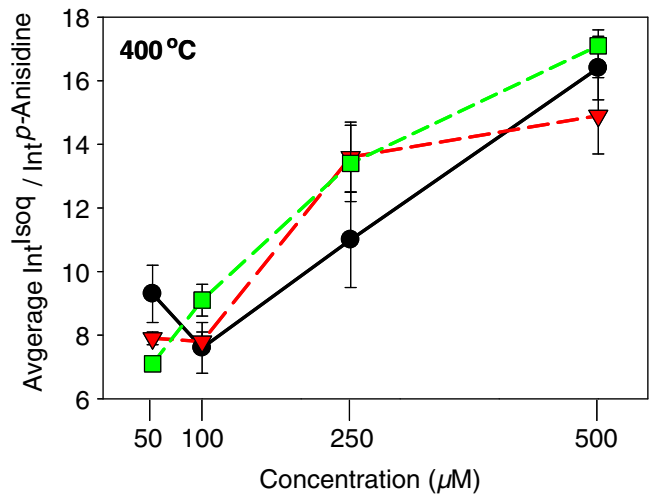

Figure 5. Effect of different proton affinities but similar boiling points on ion suppression at different sampling spots of equimolar mixtures of isoquinoline (BP: $242{ }^{\circ} \mathrm{C}$, PA $952 \mathrm{~kJ} \mathrm{~mol}^{-1}$ ) and $p$-anisidine (BP: $243{ }^{\circ} \mathrm{C}$, PA: $900 \mathrm{~kJ} \mathrm{~mol}^{-1}$ ) at DART gas temperatures of: (a) $200{ }^{\circ} \mathrm{C}$, (b) $300{ }^{\circ} \mathrm{C}$ and (c) $400{ }^{\circ} \mathrm{C}$

from 7 to $17 \times$ (Fig. $5 b$ and c). A decrease in detected protonated water cluster abundance during the experiments (Supplementary Figure S3) was observed in correlation with the increase in suppression with overall concentration. For instance, at $500 \mu \mathrm{M}$, the measured average intensities of protonated waters ranging in size from $n=7-19$ decreased compared with that at lower concentrations (Supplementary Figure S3d). Water clusters below $n=7$ were not detected due to the data acquisition range used and the ion guide $\mathrm{rf}$ voltages chosen, which did not allow detection of low $n$ clusters.
Oddly, for all three temperatures, there was a dip in the level of suppression at the equimolar concentration of $100 \mu \mathrm{M}$. In most trials, this dip was small and within the experimental error, however position B1 suffered from the largest dips. This effect cannot be explained by ion transmission or thermal dissipation effects since transmission losses should be less noticeable at higher concentrations, and because position B1 experiences the highest temperatures (Fig. 2). The dip may be attributed to fluctuations in reactive species under those conditions. For clusters with $n=7-13$, there was a slight increase in intensity for position B1 at $50 \mu \mathrm{M}$ compared with $100 \mu \mathrm{M}$ (Supplementary Figure S3a and b). The extreme dip at $300{ }^{\circ} \mathrm{C}$ may have been caused from a gas turbulence disturbance between trials.

One other interesting, but currently unexplained, phenomenon was the relative higher intensity of midsized water clusters at $n=9,11,13$, and 17 in some trials (Supplementary Figure S3). As a whole, the number of stable water cluster isomers increases exponentially with $n$ [24]. The higher abundance of specific clusters has been observed previously with DART [20], but that study showed relative higher levels of water clusters $n=3,6,9$, and 12 for various temperatures $\left(175,250\right.$, and $\left.325^{\circ} \mathrm{C}\right)$ and flow rates $\left(2,4\right.$, and $\left.6 \mathrm{Lmin}^{-1}\right)$. Although there are no significant differences in binding energies [25] and bond dissociation energies [26] for water clusters in the range $9 \leq n \leq 17$, the atmospheric pressure interface in the instrument used in this study (GIST-capillarydual ion funnel) is much different than that in previous work (cone/skimmer orifice) [20]. Therefore, we believe that the observed differences in water cluster species are an effect caused more by the ion transfer optics design and reduced pressure region architecture than the by preferential formation or reactivity of particular species.

After observing the major contributing factor to ion suppression was differences in PA rather than BP, conditions to reduce this effect were explored. The same analytes ( $p$-anisidine and isoquinoline) were used in these tests, but molar ratios were varied from $1(50 \mu \mathrm{M}$ each of $p$-anisidine

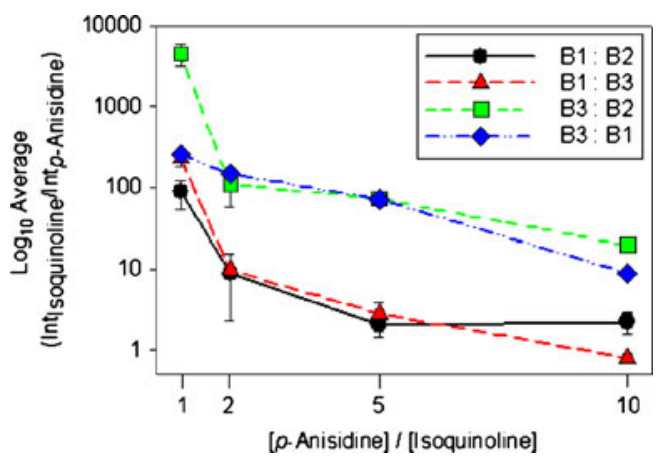

Figure 6. Ion suppression observed between isoquinoline and $p$-anisidine at different molar ratios and sampling spots for a DART gas temperature of $300{ }^{\circ} \mathrm{C}$. The legend describes the grid position pairs where isoquinoline (left) and $p$-anisidine (right) were simultaneously sampled 
and isoquinoline), to $10(500 \mu \mathrm{M}$ of $p$-anisidine and $50 \mu \mathrm{M}$ isoquinoline), such that the lower PA analyte would have an increased proportion of neutrals in the ionization region. These competition experiments were further compounded by changing the position where each individual analyte was simultaneously introduced to investigate how the relative increase of neutrals would be affected by ionization reactions in space.

As a whole, the highest suppression was at the lowest molar ratios regardless of sample position (molar ratios 1 and 2, Fig. 6). Conversely, suppression was lowest at the highest molar ratio (5 and 10, Fig. 6). The measured relative suppression did show considerable changes with respect to sample positioning. When $p$-anisidine was placed closest to the DART gas exit (B1) and isoquinoline was positioned in the middle (B2) of the ionization region, ion suppression for the molar ratios of 1,2, 5, and 10 were $88.7 \times \pm 34.8,8.8 \times \pm 6.5,2.0 \times \pm 0.6$, and $2.2 \times \pm 0.7$, respectively (black line, Fig. 6). At the same position for $p$-anisidine but with isoquinoline at $\mathrm{B} 3$, ion suppression for the molar ratios of $1,2,5$, and 10 were $231.4 \times \pm 51.2,9.6 \times \pm 0.5,2.8 \times \pm 1.1$, and $0.8 \times \pm 0.1$, respectively (red line, Fig. 6). Even though in the most favorable conditions $p$-anisidine was $7 \mathrm{~mm}$ closer to the DART source and at $10 \times$ the relative concentration level, ion suppression still existed probably due to proton scavenging by the higher PA isoquinoline from the lower PA $p$-anisidine.

A similar trend was observed when the positions were reversed such that the higher PA isoquinoline sample was continuously infused at B1 or B2 and the lower PA $p$-anisidine was injected at $\mathrm{B} 3$. Ion suppression levels were much higher in this scenario, because the higher PA molecule was closer to the DART gas exit where it would undergo the majority of reactions with protonated water clusters and scavenge protons from any $p$-anisidine protonated molecules in transit to the mass spectrometer inlet. When isoquinoline was at $\mathrm{B} 2$ and $p$-anisidine was at B3, a remarkably high suppression level of $4543.0 \times \pm$ 1406.0 was observed at an equimolar ratio (green line, Fig. 6). As the molar ratios increased to 2,5 , and 10 , the ion suppression decreased to $107.8 \times \pm 50.4,73.1 \times \pm 6.1$, and $19.4 \times \pm 2.2$, respectively. The levels of suppression were further decreased when the isoquinoline sampling position was moved closer to the DART source (B1) while $p$-anisidine remained at $\mathrm{B} 3$, thereby increasing the distance between where the two molecules were introduced. For molar ratios of $1,2,5$, and 10 ion suppression was $255.6 \times \pm 13.3,147.8 \times \pm 7.5,71.6 \times \pm 3.7$, and $8.8 \times \pm 1.2$, respectively (blue line, Fig. 6). In this sampling configuration, there was a higher likelihood that isoquinoline would ionize from reactions with protonated water clusters and not from proton scavenging. Nevertheless, there would always be suppression in the concentration range tested since isoquinoline was closer to the ionization source.

\section{Conclusions}

Spatial sensitivity and ion suppression effects are often overlooked in applications involving ambient desorption/ ionization techniques, however the results presented here demonstrate the magnitude that these can reach in certain sampling conditions. Although temperature gradients play a role in thermal desorption, concentration-dependent ion yield "hot-spots" where sensitivity was optimal may not coincide with the hottest regions in space. Additionally, differential analyte volatility plays a smaller role in ion suppression than differences in PAs. Suppression due to different PAs may be exacerbated by the spatial location of sampling. Although the experiments outlined here used continuous infusion of liquids to acquire steady-state signals, it is expected that solid samples placed within the ionization region for rapid screening purposes would show similar, if not more extensive, ion suppression due to additional flow instabilities and induced mixing in the ionization region. Future investigations of ion suppression in DART will incorporate multicomponent mixtures and higher molecular weight compounds to determine if the mass of the analytes affects ion suppression.

\section{Acknowledgments}

The authors give special thanks to Sam Mize at the College of Science machine shop for the sample block construction. The authors acknowledge support for this study by an NSF CAREER award to F.M.F.

\section{References}

1. Harris, G.A., Nyadong, L., Fernandez, F.M.: Recent Developments in Ambient Ionization Techniques for Analytical Mass Spectrometry. Analyst 133, 1297-1301 (2008)

2. Van Berkel, G.J., Pasilis, S.P., Ovchinnikova, O.: Established and Emerging Atmospheric Pressure Surface Sampling/Ionization Techniques for Mass Spectrometry. J. Mass Spectrom. 43, 1161-1180 (2008)

3. Harris, G.A., Galhena, A.S., Fernández, F.M.: Ambient Sampling/ Ionization Mass Spectrometry: Applications and Current Trends. Anal. Chem. 83, 4508-4538 (2011)

4. Cody, R.B., Laramee, J.A., Durst, H.D.: Versatile New Ion Source for the Analysis of Materials in Open Air under Ambient Conditions. Anal. Chem. 77, 2297-2302 (2005)

5. Galhena, A.S., Harris, G.A., Nyadong, L., Murray, K.K., Fernandez, F. M.: Small Molecule Ambient Mass Spectrometry Imaging by Infrared Laser Ablation Metastable-Induced Chemical Ionization. Anal. Chem. 82, 2178-2181 (2010)

6. Nyadong, L., Harris, G.A., Balayssac, S., Galhena, A.S., MaletMartino, M., Martino, R., Parry, R.M., Wang, M.D., Fernandez, F.M., Gilard, V.: Combining Two-Dimensional Diffusion-Ordered Nuclear Magnetic Resonance Spectroscopy, Imaging Desorption Electrospray Ionization Mass Spectrometry, and Direct Analysis in Real-Time Mass Spectrometry for the Integral Investigation of Counterfeit Pharmaceuticals. Anal. Chem. 81, 4803-4812 (2009)

7. Yu, S., Crawford, E., Tice, J., Musselman, B., Wu, J.T.: Bioanalysis without Sample Cleanup or Chromatography: The Evaluation and Initial Implementation of Direct Analysis in Real Time Ionization Mass Spectrometry for the Quantification of Drugs in Biological Matrixes. Anal. Chem. 81, 193-202 (2009)

8. Bevilacqua, V.L., Nilles, J.M., Rice, J.S., Connell, T.R., Schenning, A. M., Reilly, L.M., Durst, H.D.: Ricin Activity Assay by Direct Analysis in Real Time Mass Spectrometry Detection of Adenine Release. Anal. Chem. 82, 798-800 (2010) 
9. Nilles, J.M., Connell, T.R., Durst, H.D.: Quantitation of Chemical Warfare Agents Using the Direct Analysis in Real Time (Dart) Technique. Anal. Chem. 81, 6744-6749 (2009)

10. Nilles, J.M., Connell, T.R., Stokes, S.T., Dupont Durst, H.: Explosives Detection Using Direct Analysis in Real Time (Dart) Mass Spectrometry. Propellants, Explosives, Pyrotechnics 35, 446-451 (2010)

11. Harris, G.A., Kwasnik, M., Fernandez, F.M.: Direct Analysis in Real Time Coupled to Multiplexed Drift Tube Ion Mobility Spectrometry for Detecting Toxic Chemicals. Anal. Chem. 83, 1908-1915 (2011)

12. Zhou, M., Guan, W., Walker, L.D., Mezencev, R., Benigno, B.B., Gray, A., Fernandez, F.M., McDonald, J.F.: Rapid Mass Spectrometric Metabolic Profiling of Blood Sera Detects Ovarian Cancer with High Accuracy. Cancer Epidemiol. Biomarkers Prev. 19, 2262-2271 (2010)

13. Zhou, M., McDonald, J.F., Fernandez, F.M.: Optimization of a Direct Analysis in Real Time/Time-of-Flight Mass Spectrometry Method for Rapid Serum Metabolomic Fingerprinting. J. Am. Soc. Mass Spectrom. 21, 68-75 (2010)

14. Reiter, S.M., Buchberger, W., Klampfl, C.W.: Rapid Identification and Semi-Quantitative Determination of Polymer Additives by Desorption Electrospray Ionization/Time-of-Flight Mass Spectrometry. Anal. Bioanal. Chem, (2010)

15. Rothenbacher, T., Schwack, W.: Rapid Identification of Additives in Poly(Vinyl Chloride) Lid Gaskets by Direct Analysis in Real Time Ionisation and Single-Quadrupole Mass Spectrometry. Rapid Commun. Mass Spectrom. 24, 21-29 (2010)

16. Harris, G.A., Fernandez, F.M.: Simulations and Experimental Investigation of Atmospheric Transport in an Ambient Metastable-Induced Chemical Ionization Source. Anal. Chem. 81, 322-329 (2009)
17. Cody, R.B.: Observation of Molecular Ions and Analysis of Nonpolar Compounds with the Direct Analysis in Real Time Ion Source. Anal. Chem. 81, 1101-1107 (2009)

18. Shelley, J.T., Hieftje, G.M.: Ionization Matrix Effects in Plasma-Based Ambient Mass Spectrometry Sources. J. Anal. At. Spectrom. 25, 345$350(2010)$

19. Sangster, T., Spence, M., Sinclair, P., Payne, R., Smith, C.: Unexpected Observation of Ionsuppression in a Liquid Chromatography/Atmospheric Pressure Chemical Ionization Mass Spectrometric Bioanalytical Method. Rapid Commun. Mass Spectrom. 18, 1361-1364 (2004)

20. Harris, G.A., Hostetler, D.M., Hampton, C.Y., Fernandez, F.M.: Comparison of the Internal Energy Deposition of Direct Analysis in Real Time and Electrospray Ionization Time-of-Flight Mass Spectrometry. J. Am. Soc. Mass Spectrom. 21, 855-863 (2010)

21. Tabrizchi, M., Shooshtari, S.: Proton Affinity Measurements Using Ion Mobility Spectrometry. J. Chem. Thermodynamics 35, 863-870 (2003)

22. NIST Chemistry Webbook: http://webbook.nist.gov/chemistry/

23. Nicol, G., Sunner, J., Kebarle, P.: Kinetics and Kinetics and Thermodynamics of Protonation Reactions: $\mathrm{H}_{3} \mathrm{O}^{+}\left(\mathrm{H}_{2} \mathrm{O}\right)_{\mathrm{H}}+\mathrm{B}=\mathrm{Bh}^{+}$ $\left(\mathrm{H}_{2} \mathrm{O}\right)_{\mathrm{B}}+(\mathrm{H}-\mathrm{B}+1) \mathrm{H}_{2} \mathrm{O}$, Where B Is a Nitrogen, Oxygen, or Carbon Base. Int. J. Mass Spectrom. Ion Processes 84, 135-155 (1988)

24. Chang, H.C., Wu, C.C., Kuo, J.L.: Recent Advances in Understanding the Structures of Medium-Sized Protonated Water Clusters. Int. Rev. Phys. Chem. 24, 553-578 (2005)

25. Shi, Z., Ford, J.V., Wei, S., Castleman Jr., A.W.: Water Clusters: Contributions of Binding Energy and Entropy to Stability. J. Chem. Phys. 99, 8009-8015 (1993)

26. Magnera, T.F., David, D.E., Michl, J.: The First Twenty-Eight Gas-Phase Proton Hydration Energies. Chem. Phys. Lett. 182, 363-370 (1991) 